\title{
Finite-horizon dynamic optimisation when the terminal reward is a concave functional of the distribution of the final state
}

\author{
E.J. Collins \& J.M. McNamara \\ Department of Mathematics, \\ University of Bristol, \\ University Walk, \\ Bristol BS8 1TW, UK.
}

\begin{abstract}
We consider a problem similar in many respects to a finite horizon Markov decision process, except that the reward to the individual is a strictly concave functional of the distribution of the state of the individual at final time $T$. Reward structures such as these are of interest to biologists studying the fitness of different strategies in a fluctuating environment. The problem fails to satisfy the usual optimality equation and cannot be solved directly by dynamic programming. We establish equations characterising the optimal final distribution and an optimal policy $\pi^{*}$. We show that in general $\pi^{*}$ will be a Markov randomised policy (or equivalently a mixture of Markov deterministic policies) and we develop an iterative, policy improvement based algorithm which converges to $\pi^{*}$. We also consider an infinite population version of the problem, and show that the population cannot do better using a coordinated policy than by each individual independently following the individual optimal policy $\pi^{*}$.
\end{abstract}




\section{Introduction}

We consider the following sequential decision model. At each time point $t=0,1, \ldots, T<$ $\infty$ an individual is in one of a finite number of states $i \in E=\{0,1, \ldots, N\}$. The time points $t=0,1, \ldots, T-1$ form decision epochs. An individual in state $i$ has available a (possibly randomised) action from a finite set $A$. If the current state is $i$ at time $t$ and action $a$ is taken, then the state of the individual at time $t+1$ is determined by the transition probabilities $p_{i j}(a), j \in E$. (The results extend easily to state and time dependent action sets, and time dependent transition probabilities). For $t=0,1, \ldots, T$ let $x_{t}(i)$ denote the probability the individual is in state $i$ at time $t$ and let $\boldsymbol{x}_{t}$ denote the vector with components $x_{t}(i), i \in E$. The process starts with some fixed initial distribution $\boldsymbol{x}_{0}=\boldsymbol{q}$. The only reward is a final reward which is a strictly concave function $\phi$ of the final distribution $\boldsymbol{x}_{T}$. The focus of this paper is on how to characterise an optimal final distribution $\boldsymbol{x}^{*}$ which maximises $\phi(\boldsymbol{x})$ and how to characterise and compute a policy $\pi^{*}$ which achieves this final distribution $\boldsymbol{x}^{*}$.

The original motivation for considering this type of problem comes from behavioural ecology, where biologists are interested in studying the fitness of different strategies for an organism in a fluctuating environment. A number of authors have successfully treated the case where the organism only has to choose a single action (for an overview of current work see Haccou and Iwasa (1995), McNamara (1995) and Sasaki and Ellner (1995)). However, attempts to apply similar ideas to general dynamic optimisation models have often been fatally flawed. Mangel and Clark (1988) claim to perform optimisation for a problem with a fluctuating environment but their reward function is not correct for a fluctuating environment problem, and their optimisation criterion is only valid for a fixed environment. Yoshimura and Clark (1991) give an algorithm motivated by dynamic programming considerations which they incorrectly claim will directly solve the problem (see the discussion in the Example of Section 4). The first successful treatment of a general state-dependent dynamic optimisation model is provided in McNamara et al (1995) and 
one of the purposes of this present paper is to formalise and develop some of the results and techniques outlined there.

The problem we consider is very similar to a standard finite-horizon Markov decision process (MDP). For such processes the form of the optimal policy and the method of computation are well established: there is a Markov deterministic policy that is optimal and it and the corresponding optimal reward can be computed using dynamic programming (see for example Puterman (1994), Chapter 4). What makes our problem non-standard is that the (final) reward is a strictly concave function of the distribution of the final state at time $T$, and thus cannot be expressed as a function of the final state alone. This makes a large qualitative difference in that the value function for the problem fails to satisfy the principle of optimality (Bellman's equation), dynammic programming is not directly applicable and the optimal policy may in many cases be a randomised policy.

The approach we take to solving the problem is to associate a real valued reward function $R_{\pi}$ defined on $E$ with each policy $\pi$ for the individual; to associate with each policy $\pi$ a 'best response' policy $\hat{\pi}$ that maximises $E\left[R_{\pi}\left(S_{T}\right)\right]$, where $S_{T}$ is the state of the individual at time $\mathrm{T}$ and the expectation is with respect to the distribution of $S_{T}$ under the policy $\hat{\pi}$; and to show that a policy $\pi^{*}$ is optimal for the individual if and only if $\pi^{*}$ is a policy maximising $E\left[R_{\pi^{*}}\left(S_{T}\right)\right]$.

The mathematical motivation and terminology used have elements in common with work in the area of probabilistic constraints and variance in Markov decision processes (see White (1988) or Puterman (1994) Section 8.9 for surveys). In Section 3 we use results on the equivalence of different classes of policies which were originally developed in the context of probabilistic constraints by Derman and Strauch (1966) and Derman (1970). Such problems also give rise to examples where only randomised optimal policies exist (Kallenberg (1983)). Miller (1978) and Sobel (1982) give examples in the context of variance models for which the principle of optimality does not apply but do not develop any general method of solution.

The remaining sections of the paper are organised as follows. In Section 2 we motivate 
the problem we will consider by reference to an example from behavioural ecology. In Section 3 we describe the various types of polices and mixtures of policies that we will be considering. We point out that, because of equivalences between different classes of policies, there is no loss in restricting attention to Markov randomised policies or (equivalently) to mixtures of Markov deterministic policies in looking for an optimal policy. In Section 4 we develop an example to point up the fact that there will be cases where randomised policies do better than any deterministic policy and that uncritical application of dynamic programming for this type of problem may lead to entirely erroneous conclusions.

The main new results are in Sections 5, 6 and 7. In Section 5 we introduce a geometric description of the set of possible final distributions which makes it intuitively clear why, and when, randomised policies are optimal. We develop a type of optimality equation satisfied (in appropriate forms) by both the optimal final distribution and a corresponding optimal policy, and use this to characterise an optimal policy as a best response to itself within this framework. In Section 6 we use the optimality equation to motivate a policy-improvement algorithm for finding an optimal policy. This iterative algorithm works by solving linearised versions of the problem, for which dynamic programming is the correct and appropriate computational method. We explore exactly how the algorithm works and establish that a suitably modified version of the basic algorithm will always converge. A direct nonlinear programming approach to finding an optimal policy is also described. In Section 7 - again motivated by the biology - we look at a reformulated version of the problem in which we consider an infinite population of individuals each of whose actions can be coordinated under the direction of some central controller. The reward to the population is taken to be $E\left[\phi\left(\boldsymbol{X}_{T}\right)\right]$, where $\boldsymbol{X}_{T}$ is now the (possibly random) vector whose components correspond to the proportion of the population in each state at time $T$. We show that there is no advantage in using a coordinated policy, in that the reward to the population when all individuals independently use the individually optimal policy $\pi^{*}$ defined above is at least as great as that under any coordinated policy. Finally, the discussion in Section 8 contains a brief overview of the biological and computational issues involved in the iterative approach. 


\section{Biological Motivation}

In this section we motivate the problem we will consider by reference to an example from behavioural ecology. McNamara et al (1995) consider a simplified general model for finding an optimal behaviour for an organism in a large population when the environment fluctuates from year to year and cannot be predicted beforehand. In their model, each year is divided into two non-overlapping time intervals. In the first interval $[0, T]$ environmental conditions are the same each year; in the second they fluctuate from year to year. During the first time interval each individual organism is in some state $i \in E$ at times $t=0,1, \ldots, T$. At decision epochs $t=0,1, \ldots, T-1$ the organism chooses an action $a \in A$ and moves to a new state $j$ according to the transition probabilities $p_{i j}(a)$.

During this first time interval individual organisms do not reproduce; however, the state and time dependent strategy employed during the interval determines the probability of survival till time $T$ and the probability distribution of states at $T$ given survival. In the interval following $T$, population members reproduce and the state of the individual at $T$ and the ensuing environmental conditions determine the number of surviving descendants left by the individual next year. Each descendant starts the next year in state $i$ with probability $q(i)$, irrespective of what happened in the previous year.

Let $n(i, s)$ be the expected number of descendants left in a given year by an individual organism when the final state at time $T$ is $i$ and the ensuing environmental state is $s$. Now consider a genotype for which all organisms with this genotype independently follow a particular strategy $\pi$ under which the distribution of each individual at time $T$ is given by $\boldsymbol{x}^{\pi}\left(\equiv \boldsymbol{x}_{T}^{\pi}\right)$. Assume that the population of such organisms is sufficiently large for demographic stochasticity to be negligible compared with environmental stochasticity. The proportion of organisms in state $i$ at time $T$ is then given by $x^{\pi}(i)$ and the population grows by a factor

$$
r_{\pi}(s)=\Sigma_{i} n(i, s) x^{\pi}(i) .
$$

when the environmental state after time $T$ is $s$. 
In a fluctuating environment the environmental state is a random variable $S$ and the appropriate measure of fitness is the geometric mean rate of growth, or geometric mean fitness (Lewontin and Cohen, 1969). For a genotype under which organisms independently follow policy $\pi$ the geometric mean fitness is $G_{\pi}=e^{g_{\pi}}$ where $g_{\pi}=E\left[\log r_{\pi}(S)\right]$.

To see how this depends on the final distribution $\boldsymbol{x}$ let

$$
\phi(\boldsymbol{x})=E\left[\log \left(\Sigma_{i} n(i, S) x(i)\right)\right]
$$

then $\phi(\boldsymbol{x})$ is a strictly concave function of $\boldsymbol{x}$ and the genotype with the greatest geometric mean fitness is the one for which the corresponding policy maximises $\phi(\boldsymbol{x})$. Thus the problem of determining an optimal genotype corresponds precisely to the problem introduced in Section 1. 


\section{Policies and mixtures of policies}

In this section we describe the various classes of polices and mixtures of policies, and equivalences between them.

For $t=0,1, \ldots, T-1$ a decision rule $d_{t}$ specifies how the action is chosen as a function of the current history $h_{t}=\left(i_{0}, a_{0}, \ldots, i_{t-1}, a_{t-1}, i_{t}\right)$, where $i_{t}$ and $a_{t}$ denote the state and action of the individual at each time $t$. We say a decision rule is Markov if it depends only on the current state $i_{t}$ and not on the previous states and actions, otherwise it is historydependent. We say a decision rule is randomised if it specifies a probability distribution on $A$ according to which the action is chosen, and we say it is deterministic if it specifies a single action which is chosen with certainty. A policy $\pi=\left(d_{0}, d_{1}, \ldots, d_{T-1}\right)$ specifies a sequence of decision rules, where $d_{t}$ is the rule to be used at time $t$. We say a policy $\pi$ is deterministic (respectively randomised, Markov, history-dependent) if all the decision rules specified by $\pi$ are deterministic (respectively randomised, Markov, history-dependent).

We can classify the policies under consideration as history-dependent and randomised $(H R)$, Markov and randomised $(M R)$ or Markov and deterministic $(M D)$. Let $\Pi_{H R}, \Pi_{M R}$ and $\Pi_{M D}$ denote the corresponding classes of policies, then $\Pi_{M D} \subset \Pi_{M R} \subset \Pi_{H R}$.

In the regular Markov decision process framework, although $\Pi_{H R}$ is the most general class of policies one needs to consider, standard results (e.g. Puterman, 1994, Chapter 4) show that there is no loss in restricting attention to policies $\pi \in \Pi_{M D}$ when looking for an optimal policy.

This is not the case in our non-standard problem and we need to explore more carefully what kind of policies are optimal. In particular, we will find it useful to allow consideration of mixtures of policies (where we say $\mu$ is a mixture of the finite set of policies $\pi_{1}, \ldots, \pi_{K}$ with mixing probabilities $\lambda_{1}, \ldots, \lambda_{K}$ if an individual using $\mu$ makes a preliminary choice of a policy $\pi_{k}$ from the set $\left\{\pi_{1}, \ldots, \pi_{K}\right\}$ according to the respective probabilities $\lambda_{1}, \ldots, \lambda_{K}$ and then uses that policy $\pi_{k}$ throughout at decision epochs $\left.t=0,1, \ldots, T-1\right)$.

Since the reward function depends only on the final distribution $\boldsymbol{x}_{T}$, in comparing 
what can be achieved by policies in different classes we need only compare the relevant final distributions. Of course, the final distribution under any policy also depends on the initial distribution, and in what follows, everything will be assumed to be conditional on the given fixed initial distribution $\boldsymbol{q}$, though that dependence will be suppressed in our notation.

Let $X$ denote the set of all final distributions $\boldsymbol{x}_{T}$ achievable under some mixture of history-dependent randomised policies. We can think of each $\boldsymbol{x}_{T}$ as a point in $\mathbb{R}^{N+1}$, or more precisely in the $N$ dimensional simplex $\Sigma_{N}=\left\{\boldsymbol{x} \in \mathbb{R}^{N+1}: 0 \leq x(i) \leq 1, i \in\right.$ $\left.E ; \Sigma_{i} x(i)=1\right\}$. Similarly let $X_{H R}, X_{M R}$ and $X_{M D}$ denote the set of all final distributions that are attainable under policies in $\Pi_{H R}, \Pi_{M R}$ and $\Pi_{M D}$ respectively.

Since we are only interested in the distribution at final time $T$, we will henceforth use the abbreviated notation $\boldsymbol{x}^{\pi}\left(\equiv \boldsymbol{x}_{T}^{\pi}\right)$ to denote the final distribution under policy $\pi$ and similarly use $\boldsymbol{x}^{\mu}\left(\equiv \boldsymbol{x}_{T}^{\mu}\right)$ to denote the final distribution under a mixture $\mu$. If $\mu$ specifies using policies $\pi_{1}, \ldots, \pi_{K} \in \Pi_{M D}$ with respective probabilities $\lambda_{1}, \ldots, \lambda_{K}$ then $\boldsymbol{x}^{\mu}=\Sigma_{k=1}^{K} \lambda_{k} \boldsymbol{x}^{\pi_{k}}$, i.e. $\boldsymbol{x}^{\mu}$ is a convex combination of points in $X_{M D}$. The converse is also true, so convex hull $\left(X_{M D}\right)$ is precisely the set of final distributions achievable by some mixture of policies in $\Pi_{M D}$. Similarly $X=$ convex hull $\left(X_{H R}\right)$.

The following theorem (Derman and Strauch (1966), Derman (1970)) shows how $X$ can also be represented in terms of $X_{M R}$ and $X_{M D}$.

\section{Theorem 3.1.}

(i) $X=X_{M R}$

(ii) $X=$ convex hull $\left(X_{M D}\right)$.

From the theorem we see that, in looking for an optimal policy, there is no loss in restricting attention to Markov randomised policies or equivalently to mixtures of Markov deterministic policies. We also see that $X$ is a convex polytope, each of whose vertices is a point in the finite set $X_{M D}$ corresponding to the final distribution of some Markov deterministic policy. 


\section{Example}

In this section we develop an example to emphasise that dynamic programming cannot be used directly to solve this type of problem and that careless use of dynamic programming arguments may lead to entirely wrong conclusions. The example also shows that in a given problem the class of policy that is optimal may depend on the initial distribution - there may be distributions for which the only optimal policy is a deterministic policy, distributions for which the only optimal policies are randomised policies and distributions for which policies of both types are optimal.

Consider an example with the following parameters:

$E=\{0,1\}, A=\{a, b\}, \boldsymbol{q}=\left(\frac{1}{2}, \frac{1}{2}\right)$.

$p_{i j}(a)=\left(\begin{array}{rr}6 / 16 & 10 / 16 \\ 12 / 16 & 4 / 16\end{array}\right), \quad p_{i j}(b)=\left(\begin{array}{rr}1 / 16 & 15 / 16 \\ 14 / 16 & 2 / 16\end{array}\right)$.

$\phi(\boldsymbol{x})=1-x^{2}(0)-x^{2}(1)$.

Let $T=1$ and consider the choice of action at the single decision epoch $t=0$ (so a policy policy $\pi$ is determined by a single decision rule $d$ ). Since the only history is the current state, we can classify policies as either deterministic or randomised. Let $d_{1}=(a, b), d_{2}=$ $(a, a), d_{3}=(b, b)$ and $d_{4}=(b, a)$ denote the four possible deterministic rules and $\pi_{1}, \pi_{2}, \pi_{3}$ and $\pi_{4}$ denote the corresponding deterministic policies.

In standard dynamic programming, to find the final reward one would condition on the previous 'current' state (which here is at time $t=0$ ). Taking $\phi$ as the final reward function and using the usual optimality equation, we obtain the following table which seems to indicate that the optimal decision rule is $d_{2}=(a, a)$.

\begin{tabular}{cccc} 
'Current' state & Action & 'Final distribution' $\boldsymbol{x}$ & $\phi(\boldsymbol{x})$ \\
\hline 0 & $a$ & $(6 / 16,10 / 16)$ & $120 / 256^{*}$ \\
& $b$ & $(1 / 16,15 / 16)$ & $30 / 256$ \\
\hline \multirow{2}{*}{1} & $a$ & $(12 / 16,4 / 16)$ & $96 / 256^{*}$ \\
& $b$ & $(14 / 16,2 / 16)$ & $56 / 256$ \\
\hline
\end{tabular}

However, this argument is misleading, and the following table, showing the result of using the four possible deterministic decision rules, indicates that $d_{3}=(b, b)$ is the best deterministic decision rule. 


\begin{tabular}{ccc} 
Decision rule & Final distribution $\boldsymbol{x}$ & $\phi(\boldsymbol{x})$ \\
\hline$(a, a)$ & $(9 / 16,7 / 16)$ & $252 / 512$ \\
$(a, b)$ & $(10 / 16,6 / 16)$ & $240 / 512$ \\
$(b, a$ & $(13 / 32,19 / 32)$ & $247 / 512$ \\
$(b, b)$ & $(15 / 32,17 / 32)$ & $255 / 512^{*}$ \\
\hline
\end{tabular}

It may be instructive to pause for a moment and consider where the uncritical use of a dynamic programming type argument goes wrong in the above example. The final reward depends on the final distribution $\boldsymbol{x}_{1}$ where the probabilities $x_{1}(i), i \in E$, can be thought of as long run relative frequencies - either over many repetitions in the case of a single individual, or over a single repetition for each member of a large population in the behavioural ecology application. Assume that at time $t=0$ the individual uses a (possibly randomised) decision rule $d_{0}$. For each $i \in E$, this results in a distribution at time $t=1$ which is conditional on the event that the individual was in state $i$ at time $t=0$ and took action $d_{0}(i)$. The overall (final) distribution $\boldsymbol{x}_{1}$ under the decision rule $d_{0}$ is then a linear combination of these conditional distributions, weighted by the probability of being in each state at the previous time point. However, because $\phi$ is a nonlinear function, the reward from the overall final distribution is not the corresponding linear combination of the rewards from the individual conditional distributions.

Similarly, for general $T$, consider an individual using decision rules $d_{0}, d_{1}, \ldots, d_{t-1}$ up to time $t \leq T-1$. Then the optimal action for the individual in any particular state $i$ at time $t$ depends on both the relative frequency with which he would visit each other state $j$ at time $t$ (over repetitions in which he continued to use $d_{0}, \ldots, d_{t-1}$ ) and on the action he would take in $j$ (over those repetitions in which he did visit $j$ at $t$ ). In particular, this points up one of the basic flaws in the algorithm outlined by Yoshimura and Clark (1991), since their argument implicitly assumes the optimal action depends only on the current state.

We now turn to consideration of what type of policy is optimal. For finite-horizon MDP's it is well known that a deterministic policy is optimal, and here the best deterministic policy is $\pi_{3}$ with reward $510 / 1024$. In contrast, let $\pi$ be the randomised policy which 
specifies that in state 0 the individual takes action $a$ with probability $1 / 2$ (and action $b$ with probability $1 / 2$ ) and in state 1 takes action $a$ with probability $3 / 4$ (and action $b$ with probability $1 / 4)$. For the given initial distribution $x_{0}(0)=1 / 2=x_{0}(1)$ this policy results in a final distribution of $(1 / 2,1 / 2)$ with $\phi(1 / 2,1 / 2)=1 / 2$, so there is a randomised policy that does better than any deterministic policy. [In fact, any randomised policy which specifies that in state 0 the individual takes action $a$ with probability $\alpha$ and in state 1 takes action $a$ with probability $\beta$, where $5 \alpha=2 \beta+1$, results in final distribution $(1 / 2,1 / 2)$ for the initial distribution $(1 / 2,1 / 2)]$. Alternatively we can achieve this final distribution with the mixture of policies $\mu$ which specifies that we use the deterministic policy $\pi_{1}$ with probability $1 / 5$ and the deterministic policy $\pi_{3}$ with probability $4 / 5$. Thus again, there is a mixture of deterministic policies that does better than any deterministic policy.

To see what happens for a wider range of conditions, assume the initial distribution is given by $x_{0}(0)=q=1-x_{0}(1)$, where we assume $0<q<1$ to avoid degeneracy in this one stage problem. Then we find that as $q$ varies the type of policy that is optimal also varies. For $0<q \leq 4 / 11$ and for $3 / 4 \leq q<1$ the optimal policy is deterministic; for $q=6 / 13$ and for $q=2 / 3$ the optimal final distribution can be achieved either by a deterministic policy or by one of many randomised policies. For all other values of $q$ the optimal final distribution can only be achieved by one of many randomised policies. 


\section{Characterisation of optimal final distribution and optimal policies}

We have seen that the set of achievable final distributions $X$ is a convex polytope being the convex hull of the finite set $X_{M D}$. In this section we use the geometry of the situation to characterise an optimal final distribution $\boldsymbol{x}^{*}$ and an optimal policy $\pi^{*}$.

For any two points $\boldsymbol{x}_{0}$ and $\boldsymbol{x}_{1} \in X$ we define

$$
W\left(\boldsymbol{x}_{1}, \boldsymbol{x}_{0}\right)=\nabla \phi\left(\boldsymbol{x}_{0}\right) \boldsymbol{x}_{1}
$$

where we take $\boldsymbol{x}$ to be a column vector and where $\nabla \phi(\boldsymbol{x})$ is the row vector with components $\partial \phi(\boldsymbol{x}) / \partial x(i), i \in E$. Similarly, in a slight abuse of notation, for any two policies $\pi_{0}$ and $\pi_{1}$ in $\Pi_{M R}$ we define

$$
W\left(\pi_{1}, \pi_{0}\right)=W\left(\boldsymbol{x}^{\pi_{1}}, \boldsymbol{x}^{\pi_{0}}\right),
$$

where $\boldsymbol{x}^{\pi_{0}}$ and $\boldsymbol{x}^{\pi_{1}}$ are the unique points in $X$ associated with these policies.

\section{Lemma 5.1.}

(i) There is a unique point $\boldsymbol{x}^{*} \in X$ such that $\phi\left(\boldsymbol{x}^{*}\right)=\max _{\boldsymbol{x} \in X} \phi(\boldsymbol{x})$.

(ii) $\boldsymbol{x}^{*}$ is the maximising point in $X$ if and only if $W\left(\boldsymbol{x}, \boldsymbol{x}^{*}\right) \leq W\left(\boldsymbol{x}^{*}, \boldsymbol{x}^{*}\right)$ for all $\boldsymbol{x} \in X$.

(iii) $\pi^{*}$ is a policy achieving $\boldsymbol{x}^{*}$ (and hence an optimal policy) if and only if $W\left(\pi, \pi^{*}\right) \leq$ $W\left(\pi^{*}, \pi^{*}\right)$ for all $\pi \in \Pi_{M R}$.

Proof. The existence and uniqueness of $\boldsymbol{x}$ follows from the strict concavity of $\phi$ and the convexity of $X$. Furthermore, we can obtain a characterisation of $\boldsymbol{x}^{*}$ by noting that $\boldsymbol{x}^{*}$ maximises $\phi(\boldsymbol{x})$ over $X$ if and only if $\nabla \phi\left(\boldsymbol{x}^{*}\right)\left(\boldsymbol{x}-\boldsymbol{x}^{*}\right) \leq 0$ for all $\boldsymbol{x} \in X$ (Luenberger (1973), p. 119) or equivalently

$$
\nabla \phi\left(\boldsymbol{x}^{*}\right) \boldsymbol{x} \leq \nabla \phi\left(\boldsymbol{x}^{*}\right) \boldsymbol{x}^{*} \text { for all } \boldsymbol{x} \in X \text {. }
$$

Part (iii) follows directly, since each point $\boldsymbol{x} \in X$ is associated with at least one policy $\pi \in \Pi_{M R}$. 
Example 5.2 To see how these equations might be used in practice, we look at the biological example developed in Section 2. In this example

$$
\begin{aligned}
& \phi(\boldsymbol{x}) \quad=E\left[\log \left(\Sigma_{i} n(i, S) x(i)\right)\right] \\
& \nabla \phi\left(\boldsymbol{x}^{*}\right)(i)=E\left[n(i, S) / \Sigma_{j} n(j, S) x^{*}(j)\right] \\
& \text { giving } \\
& \nabla \phi\left(\boldsymbol{x}^{*}\right) \boldsymbol{x}=\Sigma_{i} x(i) E\left[n(i, S) / \Sigma_{j} n(j, S) x^{*}(j)\right] \\
& =E\left[\Sigma_{i} n(i, S) x(i) / \Sigma_{j} n(j, S) x^{*}(j)\right]
\end{aligned}
$$

and in particular $\nabla \phi\left(\boldsymbol{x}^{*}\right) \boldsymbol{x}^{*}=1$.

Applying Lemma 5.1, we have that $\boldsymbol{x}^{*}$ is optimal here if and only if

$$
E\left[\Sigma_{i} n(i, S) x(i) / \Sigma_{j} n(j, S) x^{*}(j)\right] \leq 1 \quad \text { for all } \boldsymbol{x} \in X
$$

and that a policy $\pi^{*}$ is optimal if and only if

$$
E\left[r_{\pi}(S) / r_{\pi^{*}}(S)\right] \leq 1 \quad \text { for all } \pi \in \Pi_{M R}
$$

where $r_{\pi}(s)=\Sigma_{i} n(i, s) x^{\pi}(i)$.

This extends previous characterisations for the case of a single action ((Haccou and Iwasa (1995), McNamara (1995), Sasaki and Ellner (1995)). Similar equations were derived for this example in Section 4 of McNamara et al (1995), using different methods.

Geometric considerations also enable us to classify those cases where the only optimal policy is a randomised policy and those cases where there is a deterministic policy which is optimal. Taking $X$ as fixed and considering different possible functions $\phi$, it is clear that in some cases $\boldsymbol{x}^{*}$ will be an extreme point of $X$ (a vertex of the polytope), in some cases $\boldsymbol{x}^{*}$ will be one of the other boundary points of $X$ (an interior point of one of the edges or faces of the polytope) and in some cases $\boldsymbol{x}^{*}$ will be an interior point of the polytope.

If $\boldsymbol{x}^{*}$ is a vertex then $\boldsymbol{x}^{*} \in X_{M D}$, so there is a Markov deterministic policy that achieves $\boldsymbol{x}^{*}$. Conversely, if $\boldsymbol{x}^{*}$ is not a vertex, then in general there will not be any Markov deterministic policy that achieves $\boldsymbol{x}^{*}$ and we must resort to a Markov randomised policy or a mixture of Markov deterministic policies (it is just possible - though unlikely - that 
there might happen to be a point in $X_{M D}$ that happened to coincide with $\boldsymbol{x}^{*}$ ). In addition, if $\boldsymbol{x}^{*}$ is not a vertex then there will in general be uncountably many ways of representing a policy achieving $\boldsymbol{x}^{*}$ as a mixture of policies in $\Pi_{M D}$. 


\section{Determining an optimal policy}

\section{'Best response'}

The characterisation of an optimal final distribution and an optimal policy given in Lemma 5.1 suggests the following way for updating current candidates for the optimal policy and optimal final distribution, and for confirming whether or not given candidates are optimal.

\section{Definition 6.1}

(i) Given a point $\boldsymbol{x}_{0} \in X$ we say a point $\hat{\boldsymbol{x}}_{0}$ is a best response to $\boldsymbol{x}_{0}$ if $W\left(\boldsymbol{x}, \boldsymbol{x}_{0}\right) \leq$ $W\left(\hat{\boldsymbol{x}}_{0}, \boldsymbol{x}_{0}\right)$ for all $\boldsymbol{x} \in X$.

(ii) Similarly, given a policy $\pi_{0} \in \Pi_{M R}$ we say a policy $\hat{\pi}_{0}$ is a best response to $\pi_{0}$ if $W\left(\pi, \pi_{0}\right) \leq W\left(\hat{\pi}_{0}, \pi_{0}\right)$ for all $\pi \in \Pi_{M R}$.

Thus, given a point $\boldsymbol{x}_{0}$ and corresponding policy $\pi_{0}, \hat{\pi}_{0}$ is a best response to $\pi_{0}$ if $\boldsymbol{x}^{\hat{\pi}_{0}}$ maximises $\nabla \phi\left(\boldsymbol{x}_{0}\right) \boldsymbol{x}\left(=\nabla \phi\left(\boldsymbol{x}^{\pi_{0}}\right) \boldsymbol{x}\right)$ over $\boldsymbol{x} \in X$ and we compute $\hat{\boldsymbol{x}}_{0}$ by taking $\hat{\boldsymbol{x}}_{0}=\boldsymbol{x}^{\hat{\pi}_{0}}$. From Lemma 5.1, a policy is optimal if and only it is a best response to itself.

Computing the best response is straightforward. Define the real valued function $R_{\pi_{0}}$ on $E$ by taking $R_{\pi_{0}}(i)=\nabla \phi\left(\boldsymbol{x}_{0}\right)(i)$, where $\nabla \phi\left(\boldsymbol{x}_{0}\right)(i)$ is the $i^{\text {th }}$ component of $\nabla \phi\left(\boldsymbol{x}_{0}\right)$. Then $\nabla \phi\left(\boldsymbol{x}_{0}\right) \boldsymbol{x}=\Sigma_{i} R_{\pi_{0}}(i) x(i)=E_{\boldsymbol{x}}\left[R_{\pi_{0}}\left(S_{T}\right)\right]$, where $S_{T}$ is the state of the individual at time $T$ and $E_{\boldsymbol{x}}$ denotes expectation conditioned on $S_{T}$ having distribution $\boldsymbol{x}$. Maximising $\nabla \phi\left(\boldsymbol{x}_{0}\right) \boldsymbol{x}$ is then a standard MDP with the same state space $E$, action space $A$ and transition probabilities $p_{i j}(a)$ as before, but with an expected reward criterion where the terminal reward function $R_{\pi_{0}}$ is a function of the final state alone. Thus dynamic programming can now be used to find a policy $\hat{\pi}_{0}$ which is an optimal response and, because of the way dynamic programming works, the policy $\hat{\pi}_{0}$ it computes can be chosen as a Markov deterministic policy. We can then work forward using the known initial distribution $\boldsymbol{q}$, the known transition probabilities $p_{i j}(a)$ and the known policy $\hat{\pi}_{0}$ to find the corresponding (final) distribution $\hat{\boldsymbol{x}}_{0}=\boldsymbol{x}^{\hat{\pi}_{0}}$. 


\section{Geometrical description}

Geometrical insight into how the new distribution $\hat{\boldsymbol{x}}_{0}$ is generated from $\boldsymbol{x}_{0}$ may be gained as follows. Let $\phi$ be the usual reward function and consider the surface $z=\phi(\boldsymbol{x})$ defined on $X$ (or on $\Sigma_{N}$ ). The optimal distribution $\boldsymbol{x}^{*}$ corresponds to the maximum value of $z$. Now consider the tangent hyperplane to the surface at the point $\boldsymbol{x}_{0}$. The equation of this new surface is $z=\psi_{\boldsymbol{x}_{0}}(\boldsymbol{x})=\nabla \phi\left(\boldsymbol{x}_{0}\right)\left(\boldsymbol{x}-\boldsymbol{x}_{0}\right)$. The function $\psi_{\boldsymbol{x}_{0}}(\boldsymbol{x})$ is a linear function of $\boldsymbol{x}$ which provides a local approximation to $\phi(\boldsymbol{x})$ at the point $\boldsymbol{x}_{0}$.

Points with $\nabla \phi\left(\boldsymbol{x}_{0}\right) \boldsymbol{x}=$ constant form contours in $\Sigma_{N}$ of the surface $z=\psi_{\boldsymbol{x}_{0}}(\boldsymbol{x})$, so $\hat{\boldsymbol{x}}_{0}$ lies on the highest contour of $\psi_{\boldsymbol{x}_{0}}$ which intersects $X$. If $\nabla \phi\left(\boldsymbol{x}_{0}\right) \neq \mathbf{0}$ the points at which this contour intersects $X$ will be boundary points of $X$ (if $\nabla \phi\left(\boldsymbol{x}_{0}\right)=\mathbf{0}$ then $\boldsymbol{x}_{0}=\boldsymbol{x}^{*}$ and the maximum is achieved at all points in $\boldsymbol{X}$ ). Dynamic programming selects a Markov deterministic policy as the best response policy, and the best response point associated with this policy is in $X_{M D}$. If the only point of intersection of the contour and $X$ is a single vertex, then this point in $X_{M D}$ will be the one found as the best response. If the points of intersection contain more than one vertex then any of them may be selected (or even a point in $X_{M D}$ in the interior of the convex hull of these vertices, if such a point exists).

\section{The best-response algorithm}

The problem specification for finding the best response depends through $R_{\pi_{0}}$ on the point $\boldsymbol{x}_{0}$. Different points will generate different MDP's and this approach cannot be used to find $\boldsymbol{x}^{*}$ directly since we need to know $\boldsymbol{x}^{*}$ to identify the corresponding function $R_{\pi^{*}}$. However it does motivate the following basic iterative algorithm for computing an optimal policy and the corresponding optimal final distribution.

\section{Basic Algorithm 6.2}

1. Choose some initial policy $\pi_{0}$ and compute the corresponding point $\boldsymbol{x}_{0}$.

2. Generate a sequence of policies and points $\pi_{1}, \pi_{2}, \pi_{3}, \ldots$ and $\boldsymbol{x}_{1}, \boldsymbol{x}_{2}, \boldsymbol{x}_{3}, \ldots$ by taking 
$\boldsymbol{x}_{n+1}=\hat{\boldsymbol{x}}_{n}$ and $\pi_{n+1}=\hat{\pi}_{n}, n=0,1, \ldots$.

3. Stop if $\boldsymbol{x}_{n+1}=\boldsymbol{x}_{n}$.

In this case $\boldsymbol{x}_{n}$ satisfies equation (2) since $\nabla \phi\left(\boldsymbol{x}_{n}\right) \boldsymbol{x}_{n}=\nabla \phi\left(\boldsymbol{x}_{n}\right) \boldsymbol{x}_{n+1} \geq \nabla \phi\left(\boldsymbol{x}_{n}\right) \boldsymbol{x}$ for all $\boldsymbol{x} \in X$ (by the construction of $\boldsymbol{x}_{n+1}$ ), so that $\boldsymbol{x}_{n}$ is the optimal final distribution and $\pi_{n}$ is a corresponding optimal policy.

Computational experience indicates that this basic algorithm works very well in cases where $\boldsymbol{x}^{*}$ is a vertex of $X$ and the corresponding optimal policy is a Markov deterministic policy. McNamara et al (1995) describe an example with $T=20, N=20$ and 3 possible actions, where it had no difficulty in finding the optimal policy.

However the algorithm, as it stands, only identifies Markov deterministic policies and needs some modification to identify an optimal policy in cases where $\boldsymbol{x}^{*}$ is a not vertex of $X$ and the corresponding optimal policy is a Markov randomised policy or a mixture of Markov deterministic policies. Moreover, one can not guarantee that $\phi\left(\hat{\boldsymbol{x}}_{n}\right)$ is always better that (or even as good as) $\phi\left(\boldsymbol{x}_{n}\right)$, and even in cases where the optimal point is a vertex of $X$ the unmodified algorithm may get stuck cycling between suboptimal points and never reach $\boldsymbol{x}^{*}$.

Often, a slight heuristic modification is all that is needed to ensure convergence. For example, if the best response cycles between two vertices $\boldsymbol{x}_{1}$ and $\boldsymbol{x}_{2}$, then one can break out of the cycle by finding the point $\boldsymbol{y}=\lambda \boldsymbol{x}_{1}+(1-\lambda) \boldsymbol{x}_{2}$ maximising $\phi(\boldsymbol{x})$ along the line segment joining $\boldsymbol{x}_{1}$ and $\boldsymbol{x}_{2}$ and using this as the starting point of the next iteration.

\section{Modified best-response algorithm}

We show below that it is possible to constuct a modified algorithm incorporating the bestresponse method which produces a strict improvement at each iteration, which converges to the optimal final distribution in a finite number of iterations and where the optimal policy can be identified even when it is a Markov randomised policy. The algorithm indicates at least one systematic way to proceed in cases where heuristic modifications may fail, in particular when $\boldsymbol{x}^{*}$ is not a vertex so that the corresponding optimal policy is not 
deterministic.

\section{Algorithm 6.3}

1. Choose some initial policy $\pi_{0}$ and compute the corresponding point $\boldsymbol{x}_{0}$.

2. Assume at the $r^{\text {th }}$ stage one has an $N$-dimensional polytope $P_{r}$ with known vertices $\boldsymbol{v}_{0}, \ldots, \boldsymbol{v}_{N}$ (which depend on $r$ ). Find the values $\lambda_{0}^{*}, \ldots, \lambda_{N}^{*}$ solving the nonlinear programming problem: $\max g(\boldsymbol{\lambda})=\phi\left(\lambda_{0} \boldsymbol{v}_{0}+\ldots+\lambda_{N} \boldsymbol{v}_{N}\right)$ subject to the constraints $\lambda_{j} \geq 0 j=0, \ldots, N$ and $\sum_{j} \lambda_{j}=1$. Set $\boldsymbol{p}_{r}=\lambda_{0}^{*} \boldsymbol{v}_{0}+\ldots+\lambda_{N}^{*} \boldsymbol{v}_{N}$.

3. If all the $\lambda_{j}^{*}$ are strictly positive then stop, with $\boldsymbol{x}^{*}=\boldsymbol{p}_{r}$; otherwise find the best response $\hat{\boldsymbol{p}}_{r}$ to $\boldsymbol{p}_{r}$. (Appropriate modifications are needed while building up the first polytope).

4. If $\hat{\boldsymbol{p}}_{r}$ is one of the vertices $\boldsymbol{v}_{0}, \ldots, \boldsymbol{v}_{N}$, then again stop, with $\boldsymbol{x}^{*}=\boldsymbol{p}_{r}$; otherwise use $\hat{\boldsymbol{p}}_{r}$ to replace a vertex with a zero coefficient $\lambda_{j}^{*}$ and proceed to step 2 for the $(r+1)^{\text {th }}$ stage of the iteration.

\section{Lemma 6.4}

(i) The sequence $\phi\left(\boldsymbol{p}_{0}^{*}\right), \phi\left(\boldsymbol{p}_{1}^{*}\right), \phi\left(\boldsymbol{p}_{2}^{*}\right), \ldots$ produced by Algorithm 6.3 is strictly increasing while the process continues.

(ii) The algorithm stops after a finite number of iterations.

(iii) The algorithm converges to the optimal distribution $\boldsymbol{x}^{*}$.

(iv) The algorithm provides a representation of the optimal policy.

\section{Proof}

(i) If one or more of the $\lambda_{j}^{*}$ are zero then $\boldsymbol{p}_{r}$ must be on a face $F_{r}$ of the polytope $P_{r}$. Since $\boldsymbol{p}_{r}$ maximises $\phi(\boldsymbol{x})$ on this face, the zero contour of the function $\psi \boldsymbol{p}_{r}(\boldsymbol{x})=\nabla \phi\left(\boldsymbol{p}_{r}\right)\left(\boldsymbol{x}-\boldsymbol{p}_{r}\right)$ is the hyperplane through $F_{r}$. Since $\hat{\boldsymbol{p}}_{r}$ is not one of the vertices (or more generally, since $\hat{\boldsymbol{p}}_{r}$ is not in this hyperplane), $\phi(\boldsymbol{x})$ is initially increasing as we move from $\boldsymbol{p}_{r}$ to $\hat{\boldsymbol{p}}_{r}$. Thus $\boldsymbol{p}_{r+1}$, the maximum of $\phi(\boldsymbol{x})$ over the new polytope $P_{r+1}$, must be strictly greater than $\boldsymbol{p}_{r}$. (ii) Each polytope has vertices in the finite set $X_{M D}$ so the number of distinct polytopes is finite and each point in $X$ is in some one of the distinct $N$-dimensional polytopes. Since $\phi\left(\boldsymbol{p}_{r}\right)<\phi\left(\boldsymbol{p}_{r+1}\right)$ until the process stops, no polytope is ever repeated in the sequence. Thus 
the sequence $P_{0}, P_{1}, \ldots$ is finite.

(iii) If the algorithm stops at step 3 then $\phi$ is maximised over $P_{r}$ is at the strictly interior point $\boldsymbol{p}_{r}$ and, by the strict concavity of $\phi, \boldsymbol{p}_{r}$ provides a global maximum of $\phi$. If the algorithm stops at step 4 , then the best response to $\boldsymbol{p}_{r}$ is a point on the same zero contour of $\psi \boldsymbol{p}_{r}(\boldsymbol{x})$. Thus there is no point in $X$ which does better than $\boldsymbol{p}_{r}$ in response to itself, and from Lemma $5.1 \boldsymbol{x}^{*}=\boldsymbol{p}_{r}$.

(iv)Let $\boldsymbol{v}_{0}, \ldots, \boldsymbol{v}_{N}$, be the final set of vertices, with corresponding policies $\pi_{0}, \ldots, \pi_{N}$ and weights $\lambda_{0}^{*}, \ldots, \lambda_{N}^{*}$. An optimal mixture $\mu^{*}$ of Markov deterministic policies can be constructed by taking $\mu^{*}$ to be the mixture which uses each $\pi_{j}$ with probability $\lambda_{j}^{*}$. Alternatively, it follows from Derman (1970) that an optimal Markov randomised policy $\pi^{*}$ can be found by taking $\pi^{*}$ to be the policy which takes action $a$ with probability $\alpha_{t}(i, a)$ if in state $i$ at time $t$, where the quantities

$$
\alpha_{t}(i, a)=\sum_{j} \lambda_{j}^{*} P_{\pi_{j}}\left(S_{t}=i, A_{t}=a\right) / \sum_{j} \lambda_{j}^{*} P_{\pi_{j}}\left(S_{t}=i\right)
$$

can be calculated from the known transition probabilities under each policy $\pi_{j}$ (or where $\alpha_{t}(i, \cdot)$ is an arbitrary distribution over $A$ if the denominator above is zero).

\section{Nonlinear programming}

Finally we describe an alternative approach to finding $\boldsymbol{x}^{*}$ which is based entirely on standard methods of nonlinear programming.

Introduce the variables $\{\rho(i, a, t), i \in E, a \in A, t=0,1, \ldots, T\}$, and let $\boldsymbol{q}$ be the initial state distribution. Consider the polytope $Z$ that is given by the set of feasible points of the following set of linear equalities and inequalities:

$$
\begin{gathered}
\sum_{a} \rho(i, a, 0)=q(i), \quad i \in E \\
\sum_{a} \rho(i, a, t+1)-\sum_{j} \sum_{a} \rho(j, a, t) p_{j i}(a)=0 \quad i \in E, t=0,1, \ldots, T-1, \\
\rho(i, a, t) \geq 0, \quad \sum_{i, a} \rho(i, a, t)=1, \quad t=1, \ldots, T .
\end{gathered}
$$


Given $\rho$, let $\boldsymbol{x}(\rho)$ be the vector with $i^{\text {th }}$ component $\sum_{a} \rho(i, a, T), i \in E$. Then the set $X$ of feasible final distributions is given by the polytope $X=\{\boldsymbol{x}(\rho): \rho \in Z\}$.

For $t=0,1, \ldots, T-1$ we can interpret the $\rho(i, a, t)$ as the probability of being in state $i$ and using action $a$ at time $t$ under the corresponding policy. Similar characterizations of the total occupation measure (total expected time that the system remains in some state $x$ and action $a$ is used) are well known, see e.g. Kallenberg (1983).

Nonlinear programing can now be used to find the $\rho^{*}$ which maximises $\phi(\boldsymbol{x}(\rho))$ subject to the constraints $\rho \in Z$, and an optimal final distribution is given by $\boldsymbol{x}^{*}=\boldsymbol{x}\left(\rho^{*}\right)$. Furthermore, an optimal Markov randomised policy can then be obtained from $\rho^{*}$ as follows: at time $t$, action $a$ should be used at state $i$ with probability $\alpha_{t}(i, a)=\rho^{*}(i, a, t) / \Sigma_{a^{\prime}} \rho^{*}\left(i, a^{\prime}, t\right)$ (where again $\alpha_{t}(i, \cdot)$ can be chosen as an arbitrary distribution over $A$ if the denominator is zero).

From the above characterization of $X$, one can easily show that there exists an optimal Markov randomised policy that uses at most $|E|$ randomizations. One possible way of showing this is the following. Let $\boldsymbol{x}^{*} \in X$ be an optimal solution. Consider a Linear Program where some dummy objective is to be maximized (e.g. $\Sigma_{i, a, t} \rho(i, a, t)$, which is in fact a constant), where the constraints are given by $\rho \in Z, \sum_{a} \rho(\cdot, a, T)=x^{*}(\cdot)$. Then the result is then obtained from standard arguments in Linear Programs (see e.g. Ross (1989)) 


\section{Optimal centralised or co-operative control The centralised control problem}

In the previous sections we considered a stochastic optimisation problem for an individual with state space $E$, action space $A$ and transition probabilities $\left\{p_{i j}(a)\right\}$. We found a policy $\pi^{*}$ that maximised a given strictly concave function $\phi$ of the distribution of the state of the individual at final time $T$.

The original motivation for studying this problem came from behavioural ecology. In Section 2 we considered a large population of organisms sharing the same genotype, under which each individual organism independently followed the same policy $\pi$. We noted that in this case the quantity describing the probability an individual was in state $i$ at time $T$ was exactly the same as the quantity describing the proportion of the population in state $i$ at time $T$.

Thus $\pi^{*}$ can also be interpreted as the policy maximising $\phi\left(\boldsymbol{x}_{T}\right)$, where we now take $\boldsymbol{x}_{T}$ to be the vector of population proportions at time $T$, and where the maximisation is over individually realisable policies (i.e. policies which can be implemented by each individual organism using information about their own history and not that of any other individuals in the population or of the population as a whole). For example, from equation (1) we saw that by taking $\phi(\boldsymbol{x})=E\left[\log \left(\sum_{i} n(i, S) x(i)\right)\right]$ we could identify $\pi^{*}$ with the genotype which had the greatest geometric mean fitness.

Having found the optimal individually realisable policy, it is pertinent to ask whether or not the population could do better acting in some cooperative way. For example, could a genotype that resulted in coordinated actions by the individual organisms achieve greater geometric mean fitness than genotypes under which individuals acted independently.

To address this question we will from now on consider a reformulated version of the problem, modelling a system (i.e. a population) with a central controller who is able to determine in some coordinated way the actions of each individual at each decision point. 


\section{Formulation as a Markov decision process}

In this subsection we identify the appropriate state space, action space etc. for the reformulated model.

Let $\boldsymbol{x}_{t}$ be a vector whose components denote the proportion of the population in each state at time $t$, so $x_{t}(i)$ denotes the proportion in state $i \in E$. The state of each individual in the population at each time point depends only on that individual's preceding state and action, and the final reward (to the system) depends only on the vector of final population proportions. Taken together, this means that, as far as the central controller is concerned, the state of the system at each time point can be fully described by the current vector of population proportions $\boldsymbol{x}_{t}$. We therefore take our state space to be $\mathcal{S}=\{\boldsymbol{x}: 0 \leq x(i) \leq$ $1, i \in E$ and $\left.\Sigma_{i} x(i)=1\right\}$. We assume that at time $t=0$ the system (population) starts in some given fixed initial state $\boldsymbol{q}$.

At decision epoch $t$, each individual in each state $i \in E$ has available a (possibly randomised) choice of actions from $A$. Since $A$ is finite, the overall prescription of a (randomised) action for each individual in each state $i$ is effectively the same as prescribing the proportion of individuals $\alpha_{t}(i, a)$ that are to take each action $a \in A$ for each $i \in E$, in that there is a choice of the $\alpha_{t}(i, a)$ 's which will lead to the same distribution for $\boldsymbol{x}_{t+1}$ (it is immaterial which individuals in state $i$ take which action $a$ as long as the proportions are correct). Thus the set of actions available to the controller at each decision epoch $t$ can be represented as $\mathcal{A}$, where each $\boldsymbol{\alpha}=(\alpha(i, a): i \in E, a \in A) \in \mathcal{A}$ specifies a family of distributions on $A$, one for each $i \in E$.

We assume that the controller can choose a deterministic or a randomised action from $\mathcal{A}$ at each decision epoch. If the current state of the system (population) is $\boldsymbol{x}_{t}$ with components $x_{t}(i), i \in E$ and a deterministic action $\boldsymbol{\alpha}$ is chosen, then the population dynamics are such that the system moves deterministically to state $\boldsymbol{x}_{t+1}$ at the next time point, where $x_{t+1}(j)=\Sigma_{i} x_{t}(i) \Sigma_{a} \alpha(i, a) p_{i j}(a)$ for $j \in E$. If a randomised action is chosen then corresponding expressions can be used to determine the distribution of $\boldsymbol{x}_{t+1}$. In both cases (the distribution of) $\boldsymbol{x}_{t+1}$ depends only on the current state $\boldsymbol{x}_{t}$ and the current action $\boldsymbol{\alpha}$. 
As usual, there are no costs or rewards at the decision epochs $t=0,1, \ldots, T-1$, but a final state $\boldsymbol{x}_{T}$ results in a reward of $\phi\left(\boldsymbol{x}_{T}\right)$. In this formulation, the final state under a given policy may be random rather than deterministic and we denote it by $\boldsymbol{X}_{T}$. We also need to extend our optimality criterion. The appropriate extension of $g_{\pi}$ in Section 2 to random $\boldsymbol{x}$ is to take $r_{\pi}(s)=\Sigma_{i} n(i, s) X^{\pi}(i)$ and $g_{\pi}=E\left[\log r_{\pi}(S)\right]$, where now the expectation is over the distribution of $\boldsymbol{X}^{\pi}$ and $S$. Motivated by this, we take our optimality criterion to be maximising $E\left[\phi\left(\boldsymbol{X}_{T}\right)\right]$.

\section{Optimality of an individually realisable policy}

The problem of finding the optimal centralised policy for the system can thus be formulated as a sequential decision problem with time points $t=0,1, \ldots, T$, with decision epochs $t=0,1, \ldots, T-1$, state space $\mathcal{S}$, action space $\mathcal{A}$, transition distribution as described above and initial state $\boldsymbol{q}$. The objective is to maximise $E\left[\phi\left(\boldsymbol{X}_{T}\right)\right]$. The important point is that in this formulation $\boldsymbol{X}_{T}$ is the actual state of the system at time $T$ so the problem is now in all respects a standard MDP.

We say a centralised policy for the controller is individually realisable if there is a policy $\pi$ for the individual problem such that the evolution of the system when all individuals independently use $\pi$ is the same as that under the centralised policy. The following lemma characterises the individually realisable policies.

\section{Lemma 7.1}

A policy for the central controller is individually realisable if and only if the policy specifies $d_{t}\left(\boldsymbol{x}_{t}, h_{t}\right)=\boldsymbol{\alpha}_{t}=\left(\alpha_{t}(i, a): i \in E, a \in A\right)$, where $\boldsymbol{\alpha}_{0}, \ldots, \boldsymbol{\alpha}_{T-1}$ are a sequence of actions in $\mathcal{A}$ that depend only on $t$ and not on $\boldsymbol{x}_{t}$ or $h_{t}$ (the history of the system at time $t$ ).

Proof. For policies not of this form, an individual, who knows only his own current state and history and is ignorant of the corresponding population quantities $\boldsymbol{x}_{t}$ and $h_{t}$, will not be able to identify the appropriate (randomised) action to take. Conversely, if the policy is of this form, then let $\pi$ be the policy, for the individual formulation of the problem, which specifies that an individual in state $i$ at time $t$ takes action $a$ with probability $\alpha_{t}(i, a)$. It 
can easily be verified that the behaviour of the system when all individuals independently use $\pi$ is exactly the same as under the corresponding centralised policy.

The following result shows that the population cannot do better acting cooperatively than it can do acting independently.

\section{Theorem 7.2}

Let $\pi^{*}$ be the optimal individual policy defined in Lemma 5.1. The individually realisable policy under which all individuals independently follow $\pi^{*}$ provides an optimal centralised policy.

Proof. Standard results for MDPs with general state and action spaces (see for example Bertsekas and Shreve (1978), Chapter 8) show that for the reformulated problem there is a Markov deterministic policy which is optimal in the wider class of historydependent randomised policies. This policy specifies a sequence of deterministic decision rules $d_{0}^{*}, \ldots, d_{T-1}^{*}$ under which the action $d_{t}^{*}(\boldsymbol{x})$ is taken when the state at decision epoch $t$ is $\boldsymbol{x}$, and each action specified is independent of the previous history of the process before t. Under this policy, for each initial state, the system achieves the corresponding optimal expected reward.

Let us now focus on the behaviour of the system under this optimal policy when the initial state is given by $\boldsymbol{q}$ ( $=\boldsymbol{x}_{0}^{*}$ say). This policy specifies the deterministic action $d_{0}^{*}(\boldsymbol{q})$ at time $t=0$ and under this action the system moves deterministically to a state $\boldsymbol{x}_{1}^{*}$ at time $t=1$ according to the rules given above. The policy then specifies a deterministic action $d_{1}^{*}\left(\boldsymbol{x}_{1}^{*}\right)$ at time $t=1$ and the system moves deterministically to a state $\boldsymbol{x}_{2}^{*}$. This continues until finally the system reaches a final state $\boldsymbol{x}_{T}^{*}$ which is optimal for the given initial state $\boldsymbol{q}$. For $t=0,1, \ldots, T-1$, define $\boldsymbol{\alpha}_{t}^{*}=d_{t}^{*}\left(\boldsymbol{x}_{t}^{*}\right)$. If the central controller implements the optimal policy, then the predetermined initial conditions, the deterministic (though state dependent) optimal decision rules and the deterministic system dynamics under these deterministic decision rules combine to ensure that the sequence of states and actions that result is exactly the same as if he had just implemented the the sequence of deterministic 
actions $\boldsymbol{\alpha}_{0}^{*}, \ldots, \boldsymbol{\alpha}_{T-1}^{*}$ at decision epochs $t=0,1, \ldots, T-1$.

However Lemma 7.1 shows that it is precisely policies of this latter type that can be realised by individually realisable policies. Let $\pi^{*}$ be the optimal individual policy found in the previous sections. Since the return under $\pi^{*}$ is as good as that under any other individual policy and since there is an individually realisable policy that has the same expected reward as the optimal centralised policy, the policy under which all individuals independently follow $\pi^{*}$ also provides an optimal centralised policy.

A heuristic derivation of this result that does not invoke standard theorems goes as follows. Assume (as seems reasonable) that under the appropriate conditions Theorem 3.1 can be extended from its setting of finite state and action space to the more general state and action spaces in our population control formulation. Then any final distribution which is realisable for this formulation can also be achieved by a mixture over Markov deterministic policies. Moreover, because of the deterministic dynamics (precisely as argued above), each Markov deterministic policy for the population control problem can be realised by the whole population independently following the appropriate individual policy.

The expected return under the mixture of Markov deterministic population policies is thus the same as the equivalent linear combination of returns under the corresponding individual policies. Since no individual policy has a return better than $\pi^{*}$, the expected return under any population policy is no better than the return when all individuals independently use $\pi^{*}$. 


\section{Discussion}

In Section 7 we solve the problem of controlling the behaviour of each individual in a large population so as to maximise some concave functional of the final distribution of individuals across states. We do this by showing that the optimal control is equivalent to the optimal policy for the individual control problem considered in the earlier sections.

In Section 6 we characterised the optimal policy $\pi^{*}$ for the individual control problem as a 'best response to itself'. In the population control setting of Section 7 we can interpret this as saying that $\pi^{*}$ must be an 'equilibrium' policy for the population in the following sense: if the rest of the population use $\pi^{*}$ then the overall reward to the population does not increase if some small fraction $\epsilon$ of the population changes to any other policy. Similarly, in the population control setting we can interpret a best response $\hat{\pi}_{0}$ to a given policy $\pi_{0}$ as follows: if the rest of the population uses $\pi_{0}$ and we let a fraction $\epsilon$ change to a different Markov deterministic policy, then for $\epsilon$ small the overall reward is best improved by this small fraction changing to $\hat{\pi}_{0}$.

This insight provides a biological as well as mathematical motivation for the basic iterative algorithm proposed in Section 6. However, the iterative approach can also have computational advantages over the direct nonlinear programmming approach.

It can be hard to evaluate the computational complexity of the respective approaches, but the standard nonlinear programmming formulation involves of the order of $T \times|E| \times|A|$ variables $\rho(i, a, t)$, with roughly $T \times|E|$ equality constraints and $T \times|E| \times|A|$ non-negativity constraints, and the computations involved grow rapidly with each of $T,|E|$ and $|A|$. In contrast, the best-response approach involves a sequence of iterations split into at most two parts; (i) a dynamic programming calculation whose size is linear in $T$ and $A$ and at most quadratic in $|E|$, and (ii) a nonlinear programming part (which only comes into play in those cases when the basic algorithm fails to converge) whose size is independent of $T$ and $|A|$ and involves $|E|$ variables, $|E|$ non-negativity constraints and a single equality constraint. 


\section{Acknowledgement}

We would like to thank the referee for his many helpful comments, for pointing out the direct nonlinear programmming formulation and for alerting us to some of the sources of material in Section 3. 


\section{References}

Bertsekas, D. and Shreve, S. (1978) Stochastic Optimal Control: The Discrete Time Case. Academic Press, New York.

Derman, C. (1970) Finite State Markovian Decision Processes. Academic Press, New York.

Derman, C. and Strauch, R. (1966) A note on memoryless rules for controlling sequential control processes. Ann. Math. Statist. 37, 276-278.

Haccou, P. and Iwasa, Y. (1995) Optimal mixed strategies in stochastic environments. Theor. Popul. Biol. 47, 212-243.

Kallenberg, L.C.M. (1983) Linear Programming and Finite Markov Control Problems. Mathematical Centre, Amsterdam.

Lewontin, R.C. and Cohen, D. (1969) On population growth in a randomly varying environment. Proc. Natl. Acad. Sci. USA 62, 1056-1060.

Luenberger, D.G. (1973) Introduction to Linear and Nonlinear Programming. Addison Wesley, Reading.

McNamara, J.M. (1995) Implicit frequency dependence and kin selection in fluctuating environments. Evol. Ecol. 9, 185-203.

McNamara, J.M., Webb, J.N. and Collins, E.J. (1995) Dynamic optimisation in fluctuating environments. Proc. Roy. Soc., B 261, 279-284.

Mangel, M. and Clark, C.W. (1988) Dynamic Modelling in Behavioural Ecology. Princeton University Press.

Miller, B. (1978) On dynamic programming for a stochastic Markovian process with an application to the mean variance models. Management Science 24, 199. 
Puterman, M.L. (1994) Markov Decision Processes: Discrete Stochastic Dynamic Programming. Wiley, New York.

Ross, K.W. (1989) Randomized and past-dependent policies for Markov decision processes with multiple constraints. Operations Research 37, 474-477.

Sasaki, A. and Ellner, S. (1995) The evolutionarily stable phenotype distribution in a random environment. Evolution 49, 337-350.

Sobel, M.J. (1982) The variance of discounted Markov decision processes. J. Appl. Prob. $19,774-802$.

White, D.J. (1988) Mean, variance and probabilistic criteria in finite Markov decision processes: a review. J. Optimization Theory and Applic. 56, 1-29.

Yoshimura, J. and Clark, C.W. (1991) Individual adaptations in stochastic environments. Evol. Ecol. 5, 173-192. 